\section{AB0055 HYDROXYCHLOROQUINE INHIBITS SOLUBLE CD154 PRODUCTION THROUGH CA2+ AND PKC SIGNALLING PATHWAY}

C.H. Tung, J.-M. Hsu, M.-C. Lu, N.-S. Lai. Division of Allergy, Immunology and Rheumatology, Dalin Tzu Chi Hospital, Buddhist Tzu Chi Medical Foundation, Chiayi, Taiwan, Republic of China, Chia-yi, Taiwan, Province of China

Background: Over-expression of membranous CD154 and soluble CD154 (sCD154) in T lymphocytes is important in pathogenesis of autoimmune diseases. PKC pathway induces SCD154 production through promoting shedding of membranous CD154.

Objectives: Hydroxychloroquine (HCQ) has been used in the treatment of autoimmune diseases for decades. We sought to identify the effects of HCQ on SCD154 and a possibly regulatory mechanism.

Methods: CD4 +T cells were isolated from the blood of healthy donor. After stimulated with ionomycin +PMA and various concentrations of $\mathrm{HCQ}$, concentration of sCD154 in the medium, expression of membranous CD154, $\mathrm{Ca}^{2+}$ pathway and PKC signalling pathway were assessed.

Results: $\mathrm{HCQ}$ attenuated intracellular sustained calcium storage release and membranous CD154 synthesis in activated T cells. Besides, HCQ inhibited PKC activation and subsequently shedding of membranous CD154.

Conclusions: $\mathrm{HCQ}$ inhibited production of SCD154 in activated T cells through suppressing $\mathrm{Ca}^{2+}$ and $\mathrm{PKC}$ signalling pathway. These findings provide one of the mechanistic insights into HCQ treatment.

\section{REFERENCES:}

[1] Alaaeddine N, Hassan GS, Yacoub D, Mourad W. CD154: an immunoinflammatory mediator in systemic lupus erythematosus and rheumatoid arthritis. Clin Dev Immunol. 2012:490148.

[2] Goules A, Tzioufas AG, Manousakis MN, Kirou KA, Crow MK, Routsias JG. Elevated levels of soluble CD40 ligand (SCD40L) in serum of patients with systemic autoimmune diseases. J Autoimmun 2006;26:165-71.

[3] Matthies KM, Newman JL, Hodzic A, Wingett DG. Differential regulation of soluble and membrane $\mathrm{CD} 40 \mathrm{~L}$ proteins in $\mathrm{T}$ cells. Cell Immunol. 2006;241:47-58.

[4] Wu SF, Chang CB, Hsu JM, Lu MC, Lai NS, Li C, Tung CH. Hydroxychloroquine inhibits CD154 expression in CD4+ T lymphocytes of systemic lupus erythematosus through NFAT, but not STAT5, signaling. Arthritis Res Ther 2017 Aug 9;19(1):183.

Disclosure of Interest: None declared

DOI: 10.1136/annrheumdis-2018-eular.3172

\section{AB0056 MEASUREMENT OF THE PRO-COAGULANT ACTIVITY OF MICROPARTICLES IN PATIENTS WITH INFLAMMATORY RHEUMATIC DISEASES: PROSPECTIVE STUDY}

C. Mekhail ${ }^{1}$, X. Guillot ${ }^{1}$, C. Prati ${ }^{1}$, P. Saas ${ }^{2}$, G. Mourey ${ }^{2}$, D. Wendling ${ }^{1}$.

${ }^{1}$ Rheumatology, CHRU Besançon; ${ }^{2} E F S$, Besançon, France

Background: Microparticles (MPs) are small membrane-bound vesicles that arise from activated and dying cells. Although the majority of MPs in the blood originate from platelets, all cells appear to be able to release MPs. Many studies have raised the implication of these MP in various processes: inflammation, thrombosis, angiogenesis. Previous studies reported inconsistent results in inflammatory rheumatic diseases. Studies have shown the correlation between the circulating MPs, platelet MPs and lymphocyte MPs in patients with spondyloarthritis $(\mathrm{SpA})$ compared to control patients. For rheumatoid arthritis (RA), platelet MPs levels were correlated with DAS28.

Objectives: The aim of this study was to search a possible correlation between the disease activity and the pro-coagulant property of microparticles, potential indirect marker of inflammation.

Methods: The test used (STA Procoag PPL) is a standardised automated test. Results are expressed as coagulation times (in seconds). It is a functional test that provides information on the procoagulant potential of microparticles. The microparticles supply the phospholipids expressed on their membrane surface and the test provides calcium and factor Xa necessary to initiate coagulation: the shorter the coagulation time the greater the procoagulant activity of the phospholipids being studied, suggesting a higher number of MP.

This is a prospective, single-centre study, including 39 patients with spondyloarthritis (ASAS criteria), 37 with rheumatoid arthritis (ACR criteria) and 26 control patients (healthy subjects, osteoarthritis). All patients underwent STA Procoag PPL test, and we collected medical data: disease activity (BASDAI, BASFI,
DAS28vs and DAS28crp and HAQ), biological inflammation (VS, CRP), duration of disease, and current treatment.

Results: The in vitro clotting time of serum of patients with spondyloarthritis and rheumatoid arthritis compared with controls was not significantly different $(p=0.23$ and $p=0.44$, respectively). Regarding the activity scores of inflammatory rheumatic disease: BASDAI and BASFI, DAS28esr, DAS28crp and HAQ for patients with RA, no correlation between these data and coagulation time was found; the same goes for biological inflammation (ESR, CRP), duration and type of treatment (Nonsteroidal anti-inflammatory drugs, DMARDs, biologics).

Abstract AB0056 - Table 1

\begin{tabular}{lccccc}
\hline STA PPL & Controls $(\boldsymbol{n}=\mathbf{2 6})$ & $\mathbf{S p A}(\mathrm{n}=\mathbf{3 9})$ & $\mathbf{p}$ & $\mathbf{R A}(\mathbf{n}=\mathbf{3 7})$ & $\mathbf{p}$ \\
\hline Mean \pm SD & $62,70 \pm 11,58$ & $66,48 \pm 12,77$ & $\mathrm{p}=0,23$ & $65,25 \pm 13,54$ & $\mathrm{p}=0,44$ \\
\hline BASDAI & $\mathrm{r}=0,0602$ & $\mathrm{p}=0,72$ & DAS28 esr & $r=0230$ & $p=0,17$ \\
BASFI & $r=-0,125$ & $\mathrm{p}=0,45$ & DAS28 crp & $r=0151$ & $p=0,37$
\end{tabular}

Conclusions: In this study, there is neither difference in values of procoagulant activity of MPs between inflammatory rheumatic diseases and control subjects, nor correlation with their activity scores or biological inflammation.

Disclosure of Interest: None declared

DOI: 10.1136/annrheumdis-2018-eular.2581

\section{AB0057 DECREASED IRISIN LEVEL AS RISK FACTOR OF PATHOLOGICAL FRACTURES IN RHEUMATOID ARTHRITIS PATIENTS}

D. Lavrova ${ }^{1}$, B. Zavodovsky ${ }^{1,2}$, L. Sivordova ${ }^{2}$, Y. Akhverdyan ${ }^{2}$, Y. Polyakova ${ }^{2}$, A. Yakovlev'. ${ }^{1}$ Volgograd State Medical University, Volgograd, Russian Federation; ${ }^{2}$ Research Institute for Clinical and Experimental Rheumatology, Volgograd, Russian Federation

Background: Recent studies have been suggested that adipokines and myokines may be implicated in bone metabolism and pathogenesis of osteoporosis (OP). ${ }^{1}$ Previous studies have revealed inverse correlation between irisin levels and vertebral fragility fractures, but no significant correlation was found between irisin and bone mineral density (BMD) as well as with lean weight. There is no previously published data about irisin levels in rheumatoid arthritis (RA) patients. ${ }^{2-4}$

Objectives: To investigate serum irisin levels in patients with RA.

Methods: We studied 170 people: 110 RA patients (mean age 53.58 \pm 12.32 ; hereinafter $\mathrm{M} \pm \mathrm{SD}$ ) and 60 healthy controls. All patients with RA were examined using dual-energy X-ray absorptiometry using LUNAR DPX-Pro densitometer. Osteoporotic fractures were confirmed by X-ray examination and/or by anamnesis data. All patients were passed through extensive clinical and laboratory examination, including N-terminal propeptide of procollagen type I, C-telopeptide of type I collagen, $25(\mathrm{OH})$-vitamin D concentration. Serum irisin levels were measured by ELISA (BioVender test system, Cat №RAG018R).

Results: The mean concentration of irisin in RA group was $14.48 \pm 7.07 \mathrm{mg} / \mathrm{ml}$ which was significantly lower than of healthy donors $-20.49 \pm 4.82 \mathrm{mg} / \mathrm{m}$ $(p<0,001)$. We subsequently divided all of RA patients into two groups: the first one $(n=44)$ included patients with reduced serum irisin levels (below $10.85 \mathrm{mg} /$ $\mathrm{mL}$ ), and the second one ( $\mathrm{n}=66)$ with normal irisin levels (above $10.85 \mathrm{mg} / \mathrm{ml}$ ). The first group had significantly higher activity (DAS28), increased frequency of extra-articular manifestations, disease duration more than 5 years, class III of functional joints disability and lower levels of $25(\mathrm{OH})$-vitamin D. We also observed higher incidence of pathological bone fractures in this group $(p=0,047)$. There wasn't any significant correlation between serum irisin level and BMD at any localization, lean, or fat weight. We did not reveal any difference of bone turnover markers (serum C-terminal telopeptide of type I collagen, serum N-terminal propeptide of type I procollagen (P1NP)) between these groups.

Conclusions: We have therefore revealed relationships between decreased serum irisin levels, 25(OH)-vitamin D concentration and higher incidence of pathological bone fractures in RA patients. We found no connexion between serum irisin levels and BMD at any site and body composition. We could consequently suppose, that irisin levels may reflect bone quality or increased fall risk.

\section{REFERENCES:}

[1] Palermo A, Strollo R, Maddaloni E, et al. Irisin is associated with osteoporotic fractures independently of bone mineral density, body composition or daily physical activity. Clin Endocrinol (Oxf) 2015;82(4):615-9.

[2] Boström P, Wu J, Jedrychowski MP, et al. A PGC1- $\alpha$-dependent myokine that drives brown-fat-like development of white fat and thermogenesis. Nature 2012;481(7382):463-8. 